\title{
The use of meta-analysis in infectious disease surveillance: Is it a relevant tool?
}

\author{
Antonio Toledo Jr ${ }^{[1]}$
}

[1]. Faculdade de Medicina, Universidade José do Rosário Vellano, Belo Horizonte, MG. Brasil.

\begin{abstract}
"Infectious disease surveillance is an ongoing process involving the systematic collection, analysis, interpretation and dissemination of health data. It aims to detect outbreaks early on, to monitor and analyze trends, and define public health priorities in order to reduce morbidity and mortality and achieve improved health ${ }^{1}$." Infectious diseases with a long incubation period and/or a high percentage of asymptomatic cases, such as viral hepatitis, represent a challenge for epidemiological surveillance systems. Passive surveillance, through the report of "new" cases, presents low effectiveness in these situations, generating reporting delays or a large number of underreported cases. Active surveillance, through case search, sentinel events, or epidemiological studies may be more effective, but it has a high cost and should be used for limited periods of time ${ }^{1}$. Technology evolution and dissemination of computers improved surveillance, enabling the use of secondary data and integration of different databases, and facilitating the accomplishment of meta-analysis. The next step is the incorporation of big data and artificial intelligence in the surveillance systems for infectious diseases ${ }^{2,3}$.

Gene Glass first used the term meta-analysis in 1976. It was defined as "the statistical analysis of a large collection of results from individual literature, for the purpose of integrating the
\end{abstract}

Corresponding author: Antonio Toledo Jr.

e-mail: toledoac@task.com.br

Orcid: 0000-0001-8912-2589

Received 7 January 2019

Accepted 11 January 2019 findings. " Since the method usually uses as "data" summary statistics derived from published reports of original studies, it is an analysis of a statistical analysis (thus, meta-analysis). Although it was first used to combine results of randomized clinical trials, it could be virtually used for any research topic, such as prevention, etiology, and diagnosis. The main objectives of a meta-analysis are to: (1) summarize and integrate results from a number of individual studies, (2) analyze differences in the results among studies, (3) overcome small sample sizes of individual studies to detect effects of interest and analyze endpoints that require larger sample sizes, (4) increase precision in estimating effects, (5) evaluate effects in subsets of patients, (6) determine if new studies are needed to further investigate an issue, and (7) generate new hypotheses for future studies. No doubt, it is a powerful tool to cumulate and summarize the knowledge, but it also controversial, as several conditions are critical and even small violations of these can lead to misleading conclusions. Readers must pay attention to some pitfalls and how authors manage them. Regarding the representativeness of included studies, publication bias (studies with positive results are more likely to be published), search bias, or selection bias (the last two are author-dependent) may occur. Other problems stem from the heterogeneity of the included studies' methods and results. Meta-analysis of observational studies presents particular challenges because of the inherent biases of study design and inclusion of different types of studies. They may have different endpoints, interventions, and populations. Despite all these, meta-analysis of observational studies may contribute to current knowledge, mainly in areas with scarce data ${ }^{4}$. The number of published meta-analyses on the prevalence of diseases has been increasing consistently since 2005 .

Hepatitis D virus (HDV) is a defective virus that replicates only in the presence of hepatitis B virus (HBV). It is estimated that $5 \%$ of the $\mathrm{HBV}$-infected individuals are co-infected with $\mathrm{HDV}$. Co-infection is more frequent in HBV hyperendemic areas and leads to more severe disease. Data on HDV prevalence is scarce and based on small cross-sectional studies. In this issue, Scarponi et al. ${ }^{5}$ presents a meta-analysis on HDV prevalence in 
South America. The authors carefully designed the study and properly managed the sources of limitations and methods bias. Despite the inherent bias of the study method, it is a pivotal study in South America and makes a very important contribution to the knowledge of HDV epidemiology and disease trends over the last 30 years.

For the more skeptical readers, I recall a famous phrase from George W. Comstock: "the art of epidemiologic reasoning is to draw sensible conclusions from imperfect data ${ }^{6}$."

Conflict of interest: The author declares that there is no conflict of interest.

\section{REFERENCES}

1. Teutsch SM. Considerations in planning a surveillance system. In: Teutsch SM, Churchill RE (Ed.). Principles and Practice of Public Health Surveillance. Oxford: Oxford Press, 2000.
2. Simonsen L, Gog JR, Olson D, Viboud C. Infectious Disease Surveillance in the Big Data Era: Towards Faster and Locally Relevant Systems. J Infect Dis. 2016;214(suppl 4):S380-S385.

3. Chae S, Kwon S, Lee D. Predicting Infectious Disease Using Deep Learning and Big Data. Int J Environ Res Public Health. 2018;15(8):E1596.

4. Stroup DF, Thacker SB. Meta-Analysis in Epidemiology. In: Armitage P, Colton T, eds. Encyclopedia of Biostatistics. $2^{\mathrm{a}}$ ed. Wiley, 2005.

5. Scarponi CFO, Silva RDN, Filho JAS, Guerra MRL, Pedrosa MAF, Mol MPG. Hepatitis Delta prevalence in South America: A Systematic Review and Meta-Analysis. Rev Soc Bras Med Trop. 2019:52:e20180289.

6. Comstock GW. Vaccine evaluation by case-control or prospective studies. Am J Epidemiol. 1990;131(2):205-7. 